\title{
Correlation between pregnancy and eye blindness (valsalva retinopathy)
}

\author{
Mostafa Maged Ali ${ }^{1 *}$, Laila Ezzat Abdelafatah ${ }^{2}$
}

${ }^{1}$ Department of Obstetrics and Gynaecology, Ministry of Health, Egypt

${ }^{2}$ Department of Obstetrics and Gynaecology, Fayoum University, Egypt

Received: 19 March 2021

Revised: 18 April 2021

Accepted: 19 April 2021

*Correspondence:

Dr. Mostafa Maged Ali,

E-mail: supermostafa200@yahoo.com

Copyright: (c) the author(s), publisher and licensee Medip Academy. This is an open-access article distributed under the terms of the Creative Commons Attribution Non-Commercial License, which permits unrestricted non-commercial use, distribution, and reproduction in any medium, provided the original work is properly cited.

\begin{abstract}
Eye diseases during pregnancy can be classified according to the cause of disease as the disease can be pregnancyinduced or due to presence of pre-existing eye disorder or due to normal physiological changes with pregnancy. Here, we have a case of valsalva retinopathy which demonstrates a condition resulting from increase in the intra-abdominal and intra-thoracic pressures owing to multiple bearing-downs in normal labour due to bad management of the process of normal labour. Ophthalmologists plus obstetricians play great role in prevention and diagnosing valsalva retinopathy. Prevention could be managed through curbing the patient from straining and by proper management of normal labour so pregnancy is a risk factor for valsalva retinopathy.
\end{abstract}

Keywords: Valsalva retinopathy, Pregnancy, Blindness, Eye loss, Bearing down, Straining

\section{INTRODUCTION}

Impairment of visual acuity during pregnancy is supposed to be a rare occurrence; however, ocular changes include a wider spectrum of physiologic and pathologic conditions which might present different symptoms and require different treatments. ${ }^{1}$

ocular manifestations of pregnancy can be classified or divided into three categories (physiological changespregnancy specific eye diseases-modification of preexisting eye diseases) many of pregnancy specific eye diseases can resolve post-partum. ${ }^{2}$ Valsalva retinopathy was known in $1972 .^{3}$

The reason for Valsalva retinopathy is that the enlarged uterus elevates intra-abdominal pressure and compresses the veins. These changes lead to increased intravenous pressure. As a result, potential Valsalva retinopathy can occur, Spontaneous rupture of ocular capillaries causes sudden visual loss. ${ }^{4}$

\section{CASE REPORT}

Female patient forty years old gravida six, para four, with two abortions- all her deliveries were in a normal vaginal way; no complications mentioned from patient in any of those deliveries except last one in which delivered dead nine-month old fetus due to prolonged labour. She came ten days

post-delivery. Last delivery was with midwife. Patient suffered from prolonged labour and straining and bearing down for a long time with sustained fundal pressure carried out by the midwife. In addition, midwife added uterotonics for accelerating delivery process without monitoring fetus and surely this contributed to more straining and bearing down, no history of hypertension and no history of diabetes mellitus and no history of renal diseases and no history of heart diseases and no history of eye diseases before and no history of ICU admission before and no history of convulsions no history of any thromboembolic diseases and no history of previous operations and no 
history of medications taken in purpose and no history of raising animals as cats. She is not smoker no history of viral hepatitis.

Complaint of the patient was she came ten days postdelivery she complains of painless unilateral (left eye) blindness felt twenty-four hours following delivery her baby and dull aching lower abdominal pain. During examination of the patient, her vitals were normal, her urine albumin is free, there is no pallor, no jaundice, no lower limb edema, no scars for operations, no black eye (no trauma signs), no petechiae, no vaginal bleeding, no abdominal distention, no shifting dullness, no abdominal tenderness, no fever detected. So, there was a radiologist and ophthalmologist consultations done their consultations revealed there is mild intraperitoneal free fluid and small myoma measuring $1.5 \times 1.7 \times 1.8 \mathrm{~cm}$ with no calcifications or degeneration inside it plus mildly enlarged liver measuring $18.5 \mathrm{~cm}$ with mild right-sided pleural effusion. First ophthalmology report one day after delivery shows vitreous haemorrhage and partial thickened PVD and subhyaloid haemorrhage with vitreoretinal traction and retina acoustically in place. Left sided pupil was irregular and sluggish. Left-sided eye visual acuity was $1 / 60$. But right-sided eye acuity was 6/9.

All her laboratory tests were normal including AST and ALT and bilirubin levels, serum albumin was 3,3 gram/dl and urine albumin is free. INR equals 1,2. Patient refused to do CT or MRI abdomen also she refused to do serum amylase and lipase tests. There was mild gradual improvement of vision started to develop two weeks after receiving prednisolone regimen and one ampole of dexamethasone (the challenging and the turn point) and felt by the patient forty days after delivery of the baby and she has conducted OCT eye examination of the macular area which showed macular neurosensory detachment occupying macular area and extended outside macular area as shown in Figure 1-2 in the text (left retinal detachment confirmed by ultrasound) and the previously existed vitreous haemorrhage subsided (this was the second report of ophthalmology). So, follow up regularly of the patient every two to three weeks must be done.

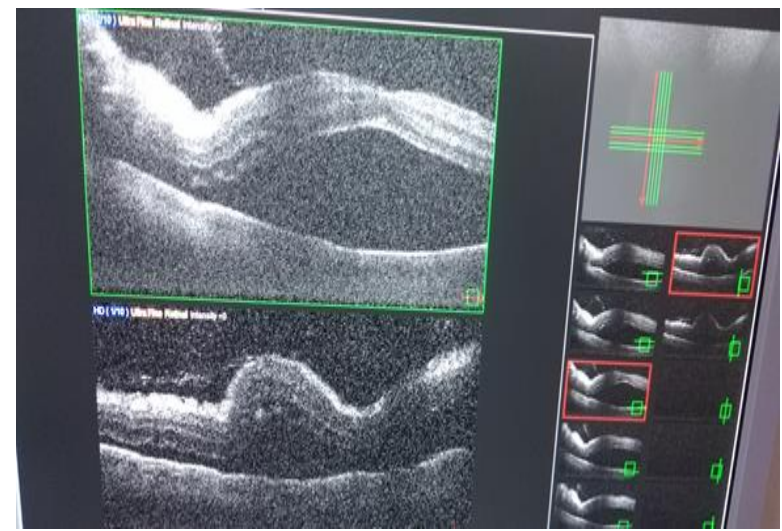

Figure 1: Oct report.

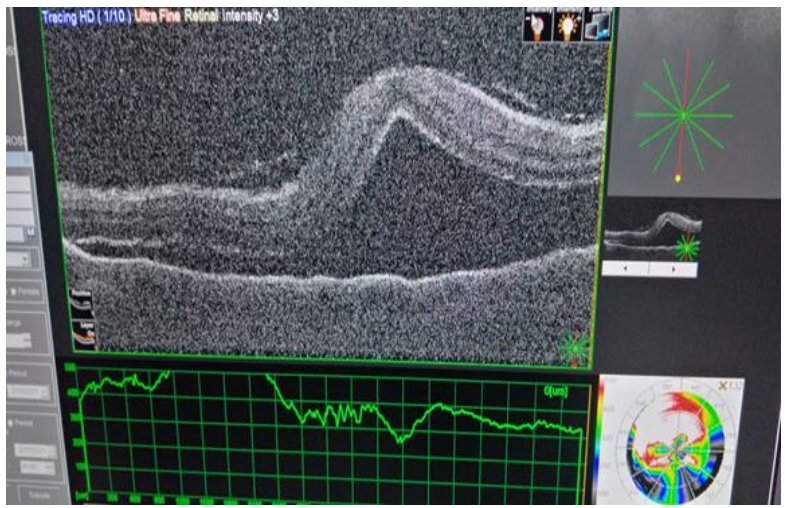

Figure 2: OCT report revealing macular neurosensory detachment occupying the macular area and extended outside macular area (left eye) as seen in images above.

\section{DISCUSSION}

Valsalva retinopathy is an induced preretinal hemorrhage that can occur in the macular area.

It usually occurs secondary to valsalva's maneuver, a forcible exhalation effort against a closed glottis, causing a sudden rise in intrathoracic pressure. ${ }^{5}$

It has previously been reported that Valsalva retinopathy can occur due to a variety of causes, such as vomiting, the lifting of a heavy object, the physical strain that occurs during child birth and general anesthesia. ${ }^{6}$ Patients with Valsalva retinopathy can be managed conservatively with careful prognosis following failed laser treatment. Ophthalmologists and obstetrician should coordinate properly and pay more attention to Valsalva retinopathic patients. $^{7}$

Prognosis is mostly good, with spontaneous resolution occurring within weeks to months after presenting symptoms, but depends where the hemorrhage is and which layer of retina involved.

Some patients may have a poor visual outcome, which has been attributed to retinal pigmentary changes in the macula. An yttrium aluminum garnet (YAG) laser has been employed in selective cases to disperse the pre-retinal hemorrhages and speed up the resolution. ${ }^{8}$ Vitrectomy is an effective and a safe procedure once spontaneous reabsorption does not occur. ${ }^{9}$

Specific advice is given to the patient where conservative management is preferred in the first instance. Patients are advised to sleep relatively upright to drain the blood inferiorly away from the maculae, avoid strenuous activities to prevent a re-bleed and to avoid constipation by consuming a fibre-rich diet and stool softeners as appropriate. $^{10}$ 
complications of valsalva retinopathy are (permanent visual loss and devastate cellular components and structure of the retina; possible toxic damage to retina due to prolonged contact of retina to hemoglobin and iron and this is irreversible damage; the operation of YAG laser membranotomy causing epiretinal membrane development).

\section{CONCLUSION}

Valsalva retinopathy is a very rare condition and selflimiting disease and can be compliacated from malmanagement of the normal labour or from straining and bearing-downs or sneezing or any form of activities which lead to increase in the intraabdominal and intrathoracic pressures. Eye examination through ophthalmologists by OCT investigation or eye ultrasound or ophthalmoscope is a must to determine the nature of eye diseases with pregnancy plus taking careful complete history from the patient is helpful as well.

Funding: No funding sources Conflict of interest: None declared

Ethical approval: Not required

\section{REFERENCES}

1. Naderan M. Ocular changes during pregnancy. J Curr Ophthalmol. 2018;30(3):202-10.
2. Omoti AE, Waziri EJM, Okeigbemen VW. A review of the changes in the ophthalmic and visual system in pregnancy. Afr J Reprod Health. 2008;12(3):185-96.

3. Oliphant H, Holmes C, Hassan A, Baddeley P. Handstand induced visual loss: Valsalva retinopathy. BMJ Case Rep. 2014;2014:2014206733.

4. Oliphant H, Holmes C, Hassan A, Baddeley P. Handstand induced visual loss: Valsalva retinopathy. BMJ Case Rep. 2014;2014206733.

5. Rubaie AK, Arevalo JF. Valsalva retinopathy associated with sexual activity. Case Rep Med. 2014; 524286.

6. Miyaki T, Kida T, Oosuka S, Fukumoto M, Sato T, Nakajima M, et al. Valsalva retinopathy induced by handstand: a case report. BMC Ophthalmol. 2020;20(1):368.

7. Li N, Zhu Z, Yi G, Li S, Han X. Valsalva Retinopathy in Twin-Pregnancy: A Case Report and Literature Review. Am J Case Rep. 2018;19:5-9.

8. Tara F, Sharifi M, Hoseini E. Valsalva retinopathy in pregnancy: a case report. BMC Res Notes. 2015;8:67.

9. Fernández MG, Navarro JC. Carmen González Castaño. J Med Case Rep. 2012;6:346.

10. Qubain WN, Khatatbeh AE. Valsalva Retinopathy during Labour: A Case Report. JRMS 2012;19(3):702 .

Cite this article as: Ali MM, Abdelafatah LE. Correlation between pregnancy and eye blindness (valsalva retinopathy). Int J Reprod Contracept Obstet Gynecol 2021;10:2059-61. 